\title{
Study on the Coupling Frequency of Double-Sided Submerged Ring-Stiffened Cylindrical Shells
}

\author{
Anbin Yu, ${ }^{1}$ Yinglong Zhao ${ }^{D},{ }^{1}$ Youqian Wang, ${ }^{2}$ and Ben Zhang ${ }^{1}$ \\ ${ }^{1}$ Institute of Noise \& Vibration, Naval University of Engineering, Wuhan 430033, China \\ ${ }^{2} 91007$ Unit of PLA, Ningbo 315122, China \\ Correspondence should be addressed to Yinglong Zhao; yinglongzhao2020@163.com
}

Received 19 August 2020; Revised 15 September 2020; Accepted 24 September 2020; Published 9 October 2020

Academic Editor: Giuseppe Ruta

Copyright (c) 2020 Anbin Yu et al. This is an open access article distributed under the Creative Commons Attribution License, which permits unrestricted use, distribution, and reproduction in any medium, provided the original work is properly cited.

Based on the Flügge theory and orthotropic theory, the acoustic vibration coupling model of ring-stiffened cylindrical shell is established by using the wave propagation method and virtual source method. And the effects of water immersion on both sides, free surface, and hydrostatic pressure on the cylindrical shell are considered in the coupling model. Muller three-point iterative method is used to solve the coupling frequency. The calculation results of degradation theory are compared with COMSOL's calculation results and experimental results, respectively, which verifies the reliability of the theoretical method. Finally, the influence of fluid load, ring rib parameters, boundary conditions, hydrostatic pressure, and free surface on the coupled vibration of ring-stiffened cylindrical shell is analyzed by an example.

\section{Introduction}

Ring-stiffened cylindrical shell [1] is a common structure in engineering, which is widely used in aircraft and naval vessels. The research on the coupled vibration characteristics of ring-stiffened cylindrical shells has always been one of the focuses of scholars. In the past, scholars only focused on the coupled vibration characteristics of ring-stiffened cylindrical shells immersed in water on single sides. With the development of engineering field, there are more and more cases that ring-stiffened cylindrical shells are immersed in water on both sides.

Therefore, it is more and more important to study the coupled vibration characteristics of ring-stiffened cylindrical shell with double sides immersed in water.

Scholars have carried out a lot of research on the coupled vibration of liquid-filled cylindrical shells. Zhang [2] used the wave method to study the vibration characteristics of cylindrical shells with fluid effects. Moeini et al. [3] used the Ritz method to consider the effect of nonuniformity of rib size on the vibration characteristics of functionally graded ring-stiffened cylindrical shells. Based on Love theory, Yang [4] studied the free vibration characteristics of hollow and liquid-filled stiffened cylindrical shells with simply supported boundary conditions. Many references [5-10] are limited to the study of the influence of single factor on the liquid-filled cylindrical shell. Liang et al. [11] comprehensively considered the effects of liquid filling factors, ring rib parameters, boundary conditions, and shell geometric parameters on the coupled vibration of liquid-filled ringstiffened cylindrical shells. Liu [12] established an acoustic vibration coupling model of a liquid-filled cylindrical shell to study the effect of hydrostatic pressure on the coupling frequency. The results show that the coupling frequency increases with the internal hydrostatic pressure.

Scholars have also carried out corresponding research on the coupled vibration of underwater immersion cylindrical shells. Chen et al. [13] calculated the elastic buckling critical load of ring-stiffened cylindrical shells under hydrostatic pressure by using the nondestructive prediction method. Based on the transfer matrix method, Cao et al. [14] studied the vibration characteristics of ring-stiffened cylindrical shells with variable thickness. Liu et al. [15] studied the influence of hydrostatic pressure on the input power flow of ring-stiffened cylindrical shells. Li and Zhang [16, 17] studied the free vibration of cylindrical shells by the wave 
method. Li [18] used the energy method and introduced the strain energy factor to discuss the interaction between the ring rib and the shell and analyzed the vibration characteristics of the ring-stiffened cylindrical shell. Liang et al. [19], respectively, studied the effects of hydrostatic pressure, cross-section size and number of ribs, and boundary conditions on the coupled vibration of underwater ring-stiffened cylindrical shells. In addition, Wang et al. [20-22] established the acoustic vibration coupling model of bare shell of finite length based on the virtual source method and image method, which can consider the influence of free surface and hydrostatic pressure.

The above studies only consider the coupled vibration characteristics of cylindrical shells with single side immersed in water such as liquid-filled cylindrical shells or underwater immersion cylindrical shells but have not studied the coupled vibration of cylindrical shells with both sides immersed in water. The research on the influence of free surface on the coupling frequency of ring-stiffened cylindrical shells is also empty. In this paper, based on the theory of Flügge and orthotropic theory, the coupling frequency equation of ringstiffened cylinder is established by using the wave propagation method. And the effects of water immersion on both sides, free surface, and hydrostatic pressure on the cylindrical shell are considered in the equation. The Muller threepoint iterative method is used to obtain the coupling frequency of the ring-stiffened cylindrical shell. The calculation results of degradation theory are compared with COMSOL's calculation results and experimental results, respectively, which verifies the reliability of the theoretical method. Finally, the influence of fluid load, ring rib parameters, boundary conditions, hydrostatic pressure, and free surface on the coupled vibration of ring-stiffened cylindrical shell is analyzed by an example.

\section{Theoretical Derivation}

The schematic diagram of ring-stiffened cylindrical shell with finite length under the condition of finite diving depth is shown in Figure 1. The length of the cylindrical shell is $L$, the radius of the neutral surface of the shell is $R$, the thickness of the shell is $h$, the center of the cross section of the cylindrical shell is $O$, the distance between the ribs is $d_{2}$, the width of the ribs is $b_{r}$, the height of the ribs is $h_{r}$, the eccentric distance of rib is $e_{2}$, and the diving depth of the cylindrical shell is $H$. As shown in Figure 1, the orthogonal coordinate system $(x, \theta, z)$ is established, where $x, \theta$, and $z$ are the axial, circumferential, and radial coordinates of the cylindrical shell, respectively.

Based on Flügge shell theory and orthotropic theory, the vibration equation of cylindrical shell can be expressed as follows [23]:

$$
\begin{array}{r}
\frac{\partial^{2} u}{\partial x^{2}}+\frac{1+\mu}{2} \frac{\partial^{2} v}{R \partial x \partial \theta}+\frac{1-\mu}{2}(1+k) \frac{\partial^{2} u}{R^{2} \partial \theta^{2}}-\mu \frac{\partial w}{R \partial x}+ \\
k\left(R \frac{\partial^{3} w}{\partial x^{3}}-\frac{1-\mu}{2} \frac{\partial^{3} w}{R \partial x \partial \theta^{2}}\right)-\frac{Q R}{B}\left(\frac{\partial^{2} u}{R^{2} \partial \theta^{2}}+\frac{\partial w}{R \partial x}+\frac{1}{2} \frac{\partial^{2} u}{\partial x^{2}}\right)=\frac{\rho \bar{h}}{B} \frac{\partial^{2} u}{\partial t^{2}} \\
\left(1+\mu_{2}\right)\left(\frac{\partial^{2} v}{R^{2} \partial \theta^{2}}-\frac{\partial w}{R^{2} \partial \theta}\right)+\frac{1+\mu}{2} \frac{\partial^{2} u}{R \partial x \partial \theta}+\frac{1-\mu}{2}(1+3 k) \frac{\partial^{2} v}{\partial x^{2}}+k\left(\frac{3-\mu}{2} \frac{\partial^{3} w}{\partial x^{2} \partial \theta}-\xi_{2} \frac{\partial^{3} w}{R^{2} \partial \theta^{3}}\right. \\
\left.-\xi_{2} \frac{\partial^{2} v}{R^{2} \partial \theta^{2}}+\eta_{2} \frac{\partial^{3} w}{R^{2} \partial \theta^{3}}+\eta_{2} \frac{\partial w}{R^{2} \partial \theta}\right)-\frac{Q}{B}\left(\frac{\partial^{2} v}{R \partial \theta^{2}}-\frac{\partial w}{R \partial \theta}\right)-\frac{Q R}{2 B} \frac{\partial^{2} v}{\partial x^{2}}=\frac{\rho \bar{h}}{B} \frac{\partial^{2} v}{\partial t^{2}} \\
\frac{\mu}{R} \frac{\partial u}{\partial x}+\left(1+\mu_{2}\right)\left(\frac{\partial v}{R^{2} \partial \theta}-\frac{w}{R^{2}}\right)+k\left[\frac{1-\mu}{2} \frac{\partial^{3} u}{R \partial x \partial \theta^{2}}-R \frac{\partial^{3} u}{\partial x^{3}}-\frac{(3-\mu)}{2} \frac{\partial^{3} v}{\partial x^{2} \partial \theta}\right. \\
-R^{2} \frac{\partial^{4} w}{\partial x^{4}}-\left(2+\eta_{t_{2}}\right) \frac{\partial^{4} w}{\partial x^{2} \partial \theta^{2}}-\left(1+\eta_{2}\right) \frac{\partial^{4} w}{R^{2} \partial \theta^{4}}-\left(2+\eta_{2}+2 \xi_{2}\right)\left(\frac{\partial^{2} w}{R^{2} \partial \theta^{2}}\right) \\
\left.-\left(1+\xi_{2}\right) \frac{w}{R^{2}}+\xi_{2} \frac{\partial^{3} v}{R^{2} \partial \theta^{3}}\right]+\frac{Q}{B}\left(\frac{\partial u}{\partial x}-\frac{\partial v}{R \partial \theta}-\frac{\partial^{2} w}{R \partial \theta^{2}}\right)-\frac{Q R}{2 B} \frac{\partial^{2} w}{\partial x^{2}}=\frac{\rho \bar{h}}{B} \frac{\partial^{2} w}{\partial t^{2}}-\frac{P_{r=R}}{B}
\end{array}
$$

where $\quad A_{2}=b_{r} h_{r}, \quad \bar{h}=h+\left(A_{2} / d_{2}\right), \quad B=E h /\left(1-\mu^{2}\right)$, $D=E h^{3} /\left(12\left(1-\mu^{2}\right)\right), \quad k=h^{2} / 12 R^{2}, \quad \mu_{2}=E A_{2} / B d_{2}$, $\chi_{2}=\left(E A_{2} e_{2}\right) /\left(R d_{2} B\right), \eta_{2}=\left(E I_{2}\right) /\left(D d_{2}\right), \eta_{t 2}=\left(G J_{2}\right) /\left(D d_{2}\right.$ ) , and $\xi_{2}=\chi_{2}\left(12 R^{2} / h^{2}\right)$. Among them, $\mu_{2}, \chi_{2}, \eta_{2}, \eta_{t 2}$, and $\xi_{2}$ are both dimensionless constants, $Q$ is the hydrostatic pressure (when the shell is filled with liquid, $Q=-\rho_{f} g H$, when the shell is submerged underwater, $Q=\rho_{f} g H$, and when the shell is immersed in water on both sides, $Q=0), \rho_{f}$ represents the density of water, $E$ represents the modulus of elasticity of shell, $G$ represents shear modulus of shell, $\mu$ 


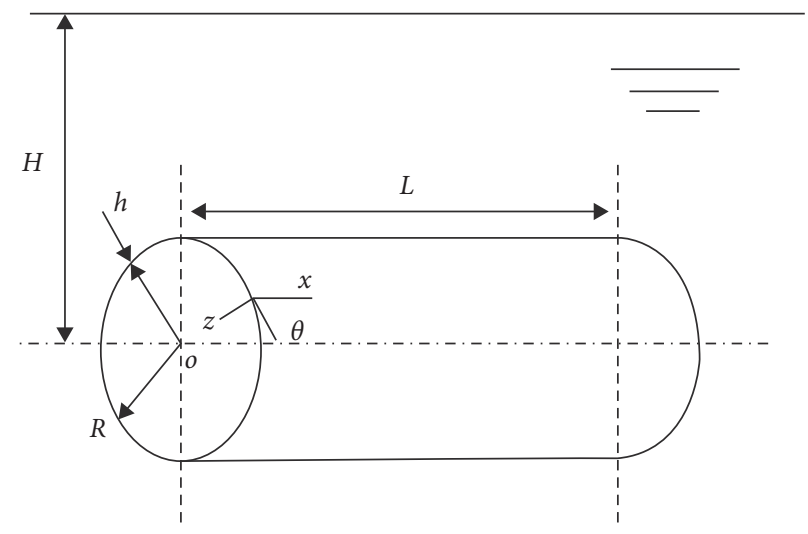

(a)

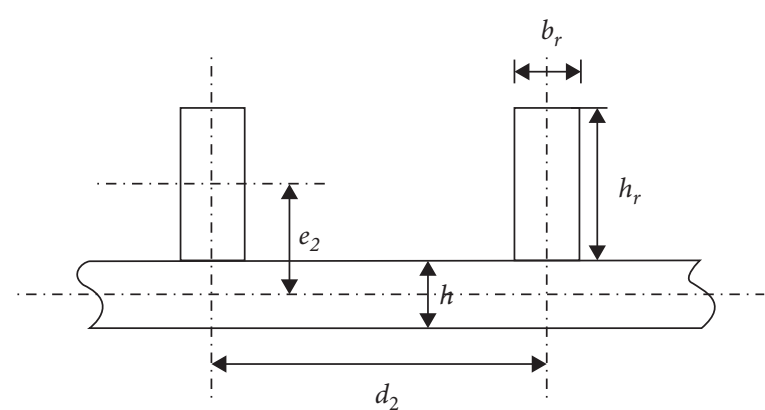

(b)

FIgure 1: Coupling model. (a) Ring-stiffened cylindrical shell. (b) Ring rib section.

represents Poisson's ratio of shell, $I_{2}$ represents moment of inertia of ring rib section to the middle plane of cylindrical shell, $J_{2}$ represents torsion constant of rib, and $P$ represents the sound pressure of fluid acting on the shell.

Based on the wave propagation method, the vibration displacement of cylindrical shell can be expressed as follows:

$$
\begin{gathered}
u=\sum_{m=1}^{+\infty} \sum_{n=0}^{+\infty} U_{m} \cos n \theta \cos k_{m} x, \\
v=\sum_{m=1}^{+\infty} \sum_{n=0}^{+\infty} V_{m} \sin n \theta \sin k_{m} x, \\
w=\sum_{m=1}^{+\infty} \sum_{n=0}^{+\infty} W_{m} \cos n \theta \sin k_{m} x .
\end{gathered}
$$

Under the condition of finite diving depth and doublesided immersion of cylindrical shell, the acoustic pressure of the fluid is mainly composed of reflected sound pressure generated by free surface and radiated sound pressure generated by liquid on both sides of cylindrical shell. Using the concept of mode attached water mass, the sound pressure of fluid can be expressed by the following formula:

$$
P=\sum_{m=1}^{+\infty} \sum_{n=0}^{+\infty} \frac{w^{2} R^{2}\left(1-\mu^{2}\right)}{E h} \sigma_{f},
$$

where $P$ represents radiation sound pressure, $w$ represents angular frequency, and $\sigma_{f}$ represents the mass density of the attached water in the $(m, n)$ order mode.

In formula (3), the mode attached water mass density is $\sigma_{f}=\sigma_{f}^{r}+\sigma_{f}^{i}$, in which $\sigma_{f}^{r}$ represents the mode attached water mass density generated by the fluid near the cylindrical shell, and $\sigma_{f}^{r}$ can be divided into three working conditions: liquid filling, underwater immersion, and double-sided immersion. The formula of mode attached water mass density under liquid filling and underwater immersion conditions is as follows:

$$
\sigma_{f}^{r}= \begin{cases}-\rho_{f} \frac{J_{n}\left(k_{r} R\right)}{k_{r} J_{n}^{\prime}\left(k_{r} R\right)}, & \text { liquid filling, } \\ \rho_{f} \frac{H_{n}^{(2)}\left(k_{r} R\right)}{k_{r} H_{n}^{(2)^{\prime}}\left(k_{r} R\right)}, & \text { underwater immersion, }\end{cases}
$$

where $J_{n}\left(k_{r} R\right)$ represents Bessel function of order $n$, $H_{n}^{(2)}\left(k_{r} R\right)$ represents Hankel function of the second kind, $k_{r}$ represents radial wave number, $k_{r}=\sqrt{\left(w / c_{0}\right)^{2}-k_{m}^{2}}, c_{0}$ represents the speed of sound in water, and $k_{m}$ represents axial half wave number, $k_{m}=(m \pi) / L$ (simply supported at both ends).

On the basis of equation (4), when the cylindrical shell is immersed in water on both sides, the mode attached water mass density can be expressed by the following formula:

$$
\sigma_{f}^{r}=\rho_{f} \frac{H_{n}^{(2)}\left(k_{r} R\right)}{k_{r} H_{n}^{(2)^{\prime}}\left(k_{r} R\right)}-\rho_{f} \frac{J_{n}\left(k_{r} R\right)}{k_{r} J_{n}^{\prime}\left(k_{r} R\right)} .
$$

$\sigma_{f}^{i}$ represents the mode attached water mass density generated by the free surface, which can be calculated by the virtual source method [24]. The result is as follows:

$$
\sigma_{f}^{i}=\rho_{f} \sum_{a=0}^{\infty} \frac{(-1)^{a+1} \Delta J_{n}\left(k_{r} R\right)}{k_{r} H_{n}^{(2)^{\prime}}\left(k_{r} R\right)} .
$$

By substituting formula (2) into formula (1) and combining formulae (3)-(6), the coupled system equation is as follows:

$$
\left[\begin{array}{ccc}
L_{11} & L_{12} & L_{13} \\
L_{21} & L_{22} & L_{23} \\
L_{31} & L_{32} & L_{33}+F L
\end{array}\right]\left[\begin{array}{c}
U_{m} \\
V_{m} \\
W_{m}
\end{array}\right]=\left\{\begin{array}{l}
0 \\
0 \\
0
\end{array}\right\},
$$

where $L_{i j}(i, j=1,2,3)$ is in the appendix, and $F_{L}$ represents the acoustic load term of fluid action which is expressed as follows: 


$$
F_{L}=\frac{w^{2} R^{2}\left(1-\mu^{2}\right)}{E h}\left(\sigma_{f}^{r}+\sigma_{f}^{i}\right)
$$

While $F_{L}=0$, the frequency of cylindrical shell in vacuum can be solved. Since equation (8) is a transcendental equation, the Muller three-point iterative method is used to solve the coupling frequency.

\section{Method Validation}

In order to verify the reliability of the theoretical method, the numerical results are compared with COMSOL's calculation results and experimental results. The model in COMSOL uses the sound-shell coupling element, and the radius of the calculation domain is three times the length of the cylindrical shell. In order to simulate the real scattering, spherical wave radiation is used on the surface of the sphere, and the maximum cell size is set to $1 / 5$ of the minimum wavelength. The specific parameters of the acoustic vibration coupling model of ring-stiffened cylindrical shell are shown in Table 1.

Table 2 shows the degradation results of the natural frequency of the double-sided water immersion bare cylindrical shell without considering the free surface and ring rib. The accuracy of the acoustic vibration coupling model proposed in this paper is verified by comparing with the calculation results of COMSOL.

In Table 3, the degradation calculation results of the natural frequency of double-sided submerged ring-stiffened cylindrical shell without considering free surface are calculated. By comparing with COMSOL's calculation results, the reliability of the natural frequency calculation method of double-sided submerged ring-stiffened cylindrical shell is verified.

In Table 4, the degradation results of natural frequencies of bare cylindrical shells immersed in water on single sides without considering ring ribs are calculated. The accuracy of the numerical method is verified by comparing with the experimental results in the literature [24].

\section{Numerical Analysis and Calculation}

4.1. Influence of Fluid Loading. The frequency characteristics of bare cylindrical shell and ring-stiffened cylindrical shell under vacuum, liquid filling, underwater immersion, and double-sided immersion are analyzed, respectively. The singlesided submerged condition is used for liquid filling and underwater immersion. The geometric parameters selected are shown in Table 1, which are used in subsequent calculation.

Figures 2 and 3 show the frequency curves of two kinds of shells at different axial half wave number $m$. It can be seen that the variation of circumferential wave number is similar between ring-stiffened cylindrical shell and bare cylindrical shell under four working conditions. When the circumferential wave number is small, the influence of axial half wave number on frequency characteristics is obvious. When the circumferential wave number is large, the influence of axial half wave number on frequency characteristics can be ignored. At the same time, it can be seen that under different modes, the order of modal frequencies size under different
TABLE 1: Coupling parameters of ring-stiffened cylindrical shell.

\begin{tabular}{lcc}
\hline Shell & Length $L(\mathrm{~m})$ & 1.284 \\
& Radius $R(\mathrm{~m})$ & 0.18 \\
& Thickness $h(\mathrm{~m})$ & 0.003 \\
& Rib height $h_{r}(\mathrm{~m})$ & 0.011 \\
& Rib width $b_{r}(\mathrm{~m})$ & 0.00621 \\
& Number and type of ribs & $N=19$, external rib \\
\hline Shell material & Density $\rho\left(\mathrm{kg} \cdot \mathrm{m}^{-3}\right)$ & 7850 \\
& Poisson's ratio $\mu$ & 0.3 \\
& Elastic modulus $E(\mathrm{~Pa})$ & $2.06 \times 10^{11}$ \\
\hline Fluid & Density $\rho_{f}\left(\mathrm{~kg} \cdot \mathrm{m}^{-3}\right)$ & 1025 \\
& Sound velocity $c_{f}\left(\mathrm{~m}^{-3} \mathrm{~s}^{-1}\right)$ & 1460 \\
\hline
\end{tabular}

TABLE 2: Natural frequencies of double-sided water immersion bare cylindrical shell.

\begin{tabular}{lccc}
\hline $\begin{array}{l}\text { Modal } \\
\text { order }\end{array}$ & $\begin{array}{c}\text { Computational results } \\
(\mathrm{Hz})\end{array}$ & $\begin{array}{c}\text { COMSOL's results } \\
(\mathrm{Hz})\end{array}$ & Error \% \\
\hline$(1,2)$ & 74.4 & 74.7 & -0.40 \\
$(1,3)$ & 83.8 & 84.5 & -0.83 \\
$(1,4)$ & 157.8 & 158.2 & -0.25 \\
$(2,3)$ & 166.3 & 169.0 & -1.60 \\
$(2,4)$ & 188.4 & 190.9 & -1.31 \\
\hline
\end{tabular}

TABle 3: Natural frequencies of double-sided water immersion ring-stiffened cylindrical shell.

\begin{tabular}{lccc}
\hline $\begin{array}{l}\text { Modal } \\
\text { order }\end{array}$ & $\begin{array}{c}\text { Computational results } \\
(\mathrm{Hz})\end{array}$ & $\begin{array}{c}\text { COMSOL's results } \\
(\mathrm{Hz})\end{array}$ & Error \% \\
\hline$(1,2)$ & 117.8 & 121.5 & -3.04 \\
$(1,3)$ & 321.7 & 322.8 & -0.33 \\
$(1,4)$ & 675.3 & 683.3 & -1.18 \\
$(2,3)$ & 346.2 & 358.7 & -3.47 \\
$(2,4)$ & 679.0 & 703.0 & -3.41 \\
\hline
\end{tabular}

TABLE 4: Natural frequencies of bare cylindrical shells near free surface $(H=0.21 \mathrm{~m})$.

\begin{tabular}{lccc}
\hline Modal order & Exp $(\mathrm{Hz})$ & Comp $(\mathrm{Hz})$ & Error \% \\
\hline$(1,2)$ & 101.6 & 100.1 & -1.48 \\
$(1,3)$ & 113.4 & 109.6 & -3.35 \\
$(1,4)$ & 203 & 204.4 & 0.69 \\
$(2,3)$ & 220.5 & 218.0 & -1.13 \\
$(2,4)$ & 243.6 & 244.3 & 0.29 \\
\hline
\end{tabular}

working conditions is vacuum, single-sided immersion, and double-sided immersion conditions, which indicates that the fluid load increases the added mass of the shell and reduces the modal frequency of the shell, and the fluid load effect under the double-sided immersion condition is more obvious than that under the single-sided immersion condition. However, for the single-sided submerged condition, the modal frequency of submerged condition is slightly larger than that of liquid-filled condition when $n=1$, and the frequency is considered to be equivalent in the whole range of circumferential wave number.

Figure 4 shows the coupling frequency ratio curves between double-sided water immersion and liquid filled under different circumferential wave numbers. It can be seen 


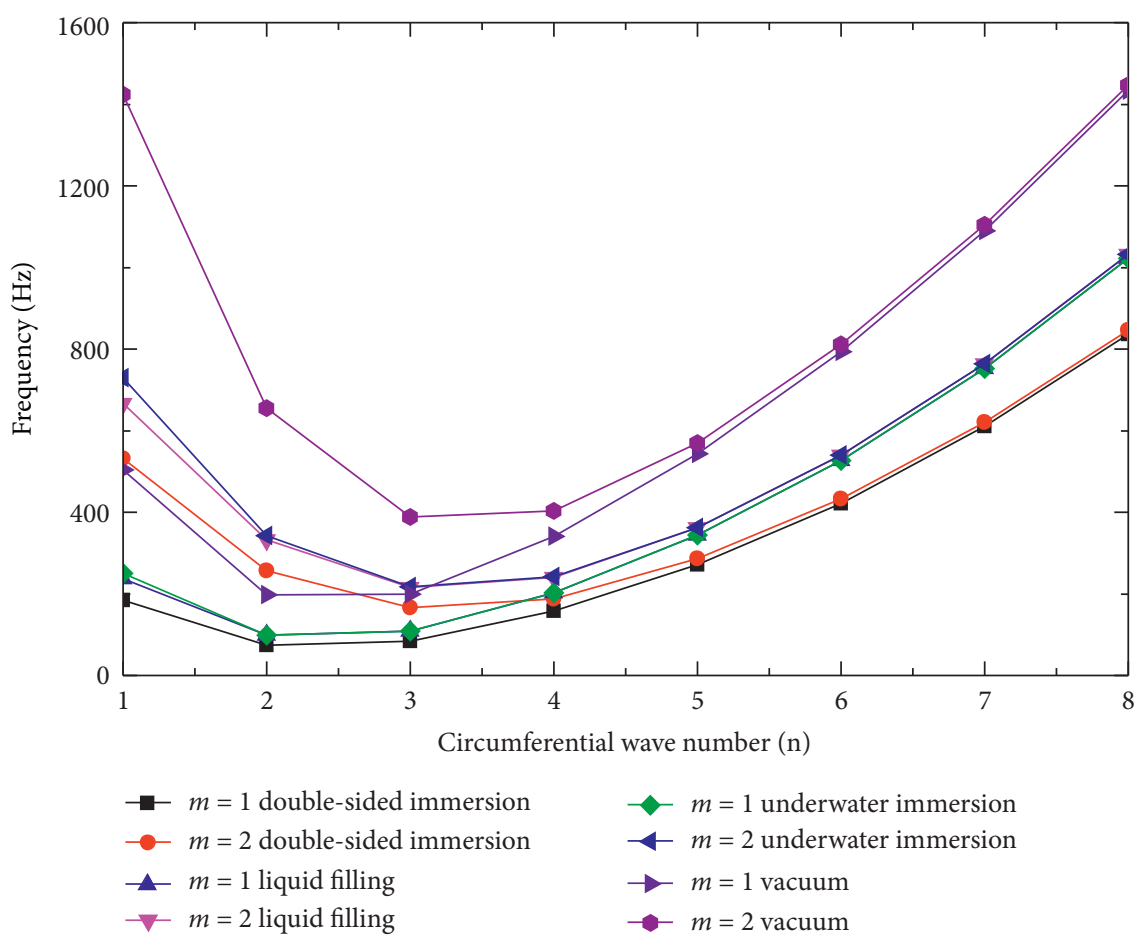

FIGURE 2: Frequency curves of bare cylindrical shell.

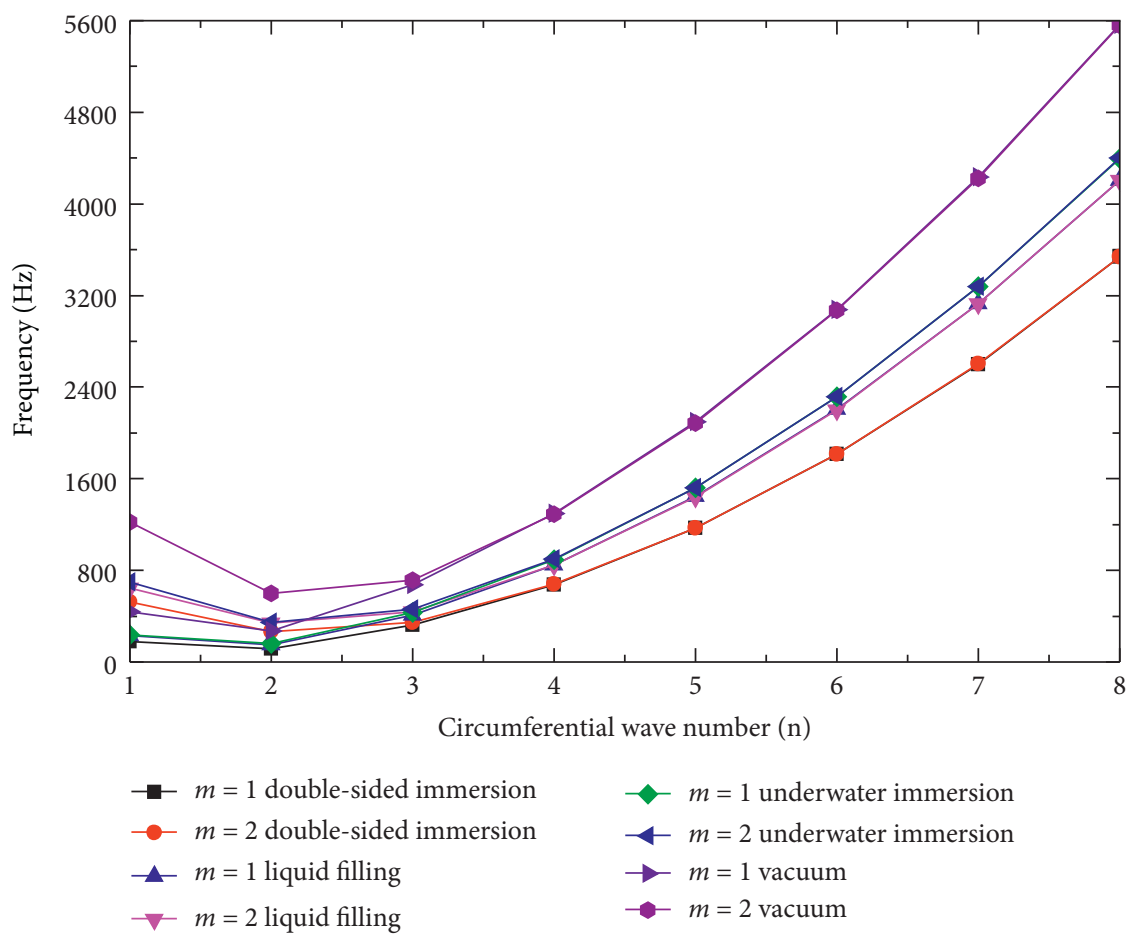

FiguRE 3: Frequency curves of ring-stiffened cylindrical shells.

from the figure that the coupling frequency ratio of ringstiffened cylindrical shell is greater than that of bare cylindrical shell, which indicates that the effect of stiffened on coupled vibration is obvious. At the same time, it can be seen that when the circumferential wave number is small, the influence of axial half wave number on the ratio of doublesided immersion coupling frequency to liquid-filled coupling frequency is obvious; when the circumferential wave number is large, the influence of axial half wave number on frequency ratio can be ignored. 


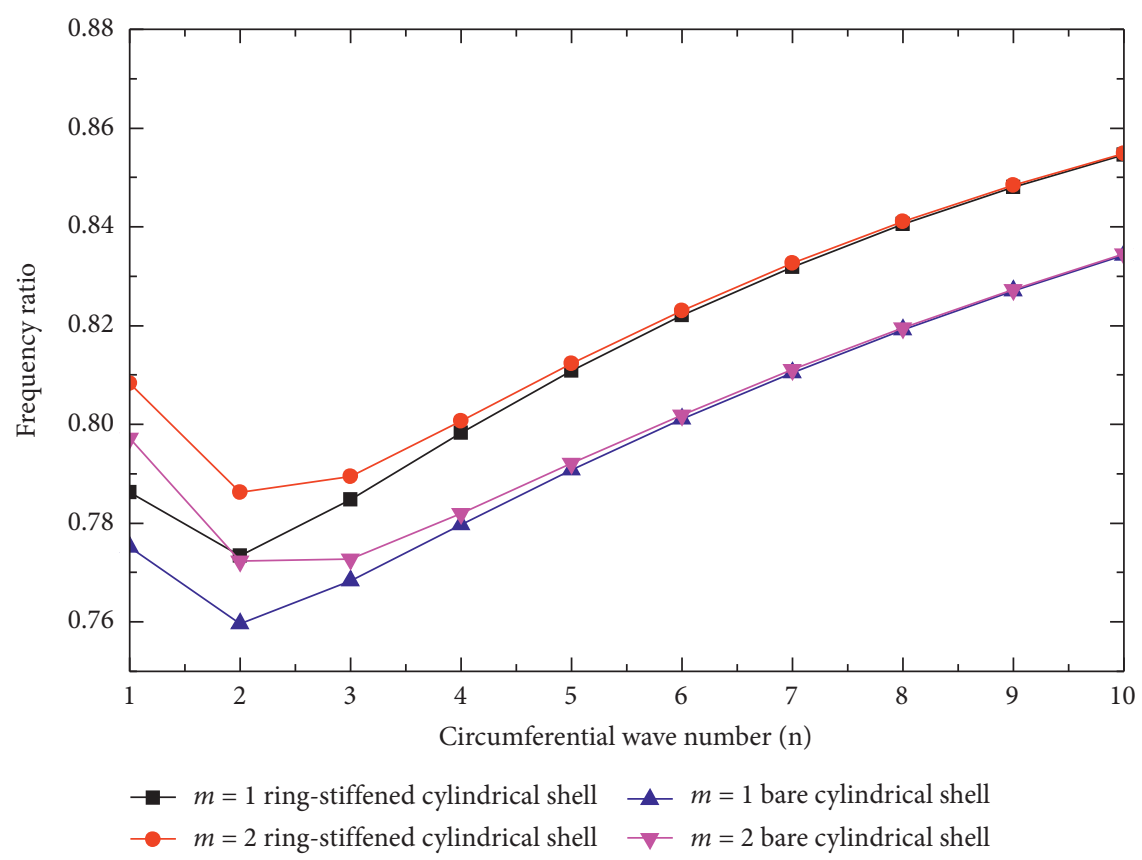

Figure 4: Variation curves of the ratio of coupling frequency of cylindrical shell.

4.2. Influence of Ring Rib. Figure 5 shows the coupling frequency curves of double-sided water immersion ring-stiffened cylindrical shells at different rib heights, and the influence of ring rib size on the frequency of ring-stiffened cylindrical shell is discussed. Take $h_{r}=0.0055 \mathrm{~m}, 0.00825 \mathrm{~m}, 0.011 \mathrm{~m}$, and $0.01375 \mathrm{~m}$, respectively. It can be seen from the figure that the coupling frequency of the cylindrical shell increases with the increase of the rib height, and the amplitude increases obviously with the circumferential wave number $n$; the influence of the variation of the rib height on the coupling frequency is mainly manifested in the case of large circumferential wave number; when the circumferential wave number $n=1$, the vibration is equivalent to the beam type vibration of the structure, and the height of the rib plays an additional role in adding mass to the stiffened cylinder shell. The frequency contribution of the shell is small.

Figure 6 shows the coupling frequency curves of doublesided water immersion ring-stiffened cylindrical shell with different number of ribs and discusses the influence of the number of ring ribs on the frequency of ring-stiffened cylindrical shell. Take $N=0,9,14,19$, and 24 , respectively. It can be seen from the figure that with the increase of the number of ribs, the coupling frequency of the cylindrical shell increases, and the amplitude increases significantly with the circumferential wave number $n$; compared with the height of the ribs, the number of ribs has a relatively weak contribution to the coupling frequency.

4.3. Influence of Boundary Conditions. Figure 7 shows the variation curves of coupling frequency with circumferential wave number for liquid-filled ring-stiffened cylindrical shell and double-sided water immersion ringstiffened cylindrical shell under four boundary conditions. It can be seen from the figure that the frequency

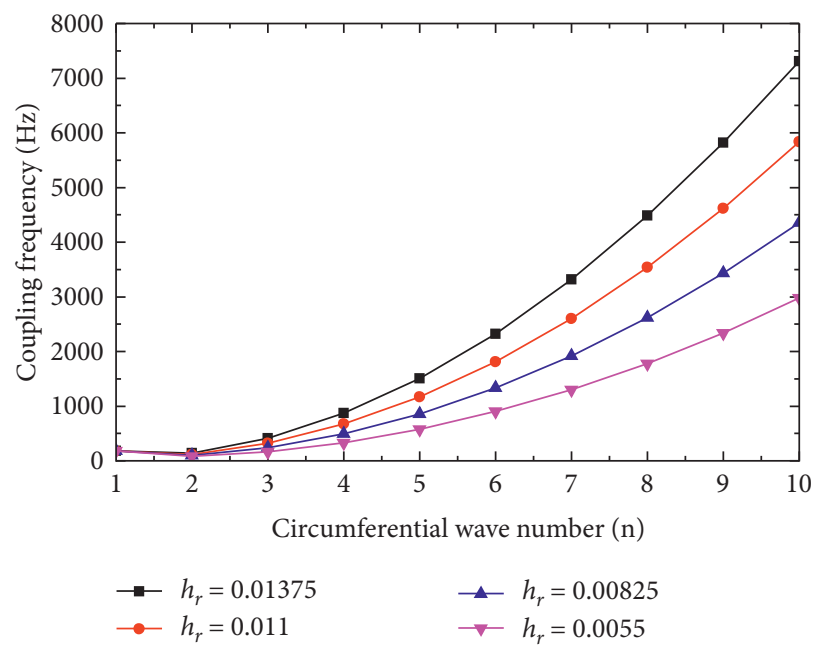

Figure 5: Coupling frequency curves of different rib heights.

variation rules of the ring-stiffened cylindrical shell under the four boundary conditions under the two working conditions are relatively consistent. When the axial wave number is small, the boundary conditions have a greater impact on the frequency of the ring-stiffened cylindrical shell, and the coupling frequency is the largest when the two ends are fixed. When the axial wave number is large, the influence of boundary conditions on the frequency can be ignored, but the coupling frequency of the liquid-filled cylindrical shell is still greater than that of the doublesided water immersion ring rib cylindrical shell, that is, the fluid loading effect still exists.

4.4. Influence of Hydrostatic Pressure. In order to study the change of coupling frequency of ring-stiffened cylindrical 


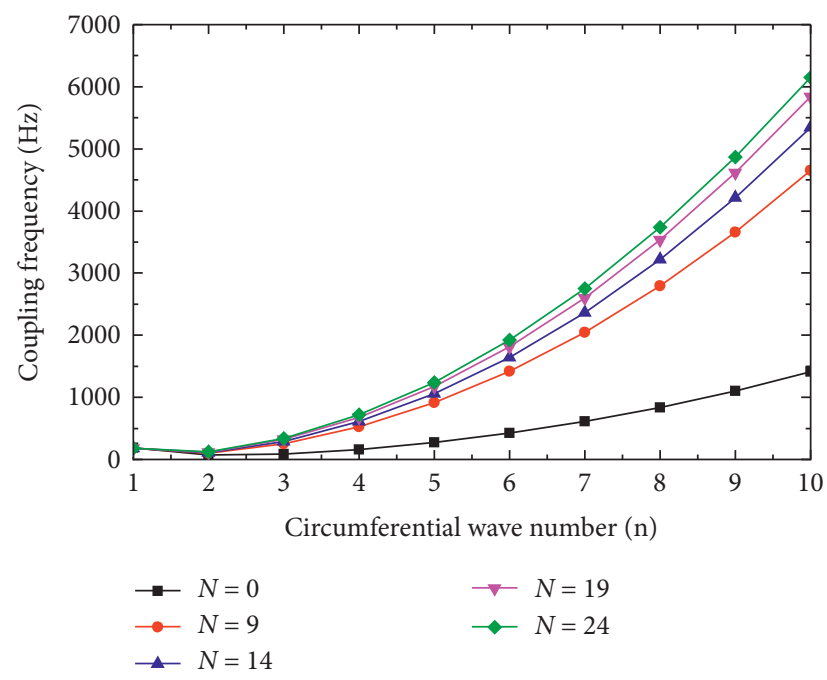

FigURE 6: Coupling frequency curves of different number of ring ribs.

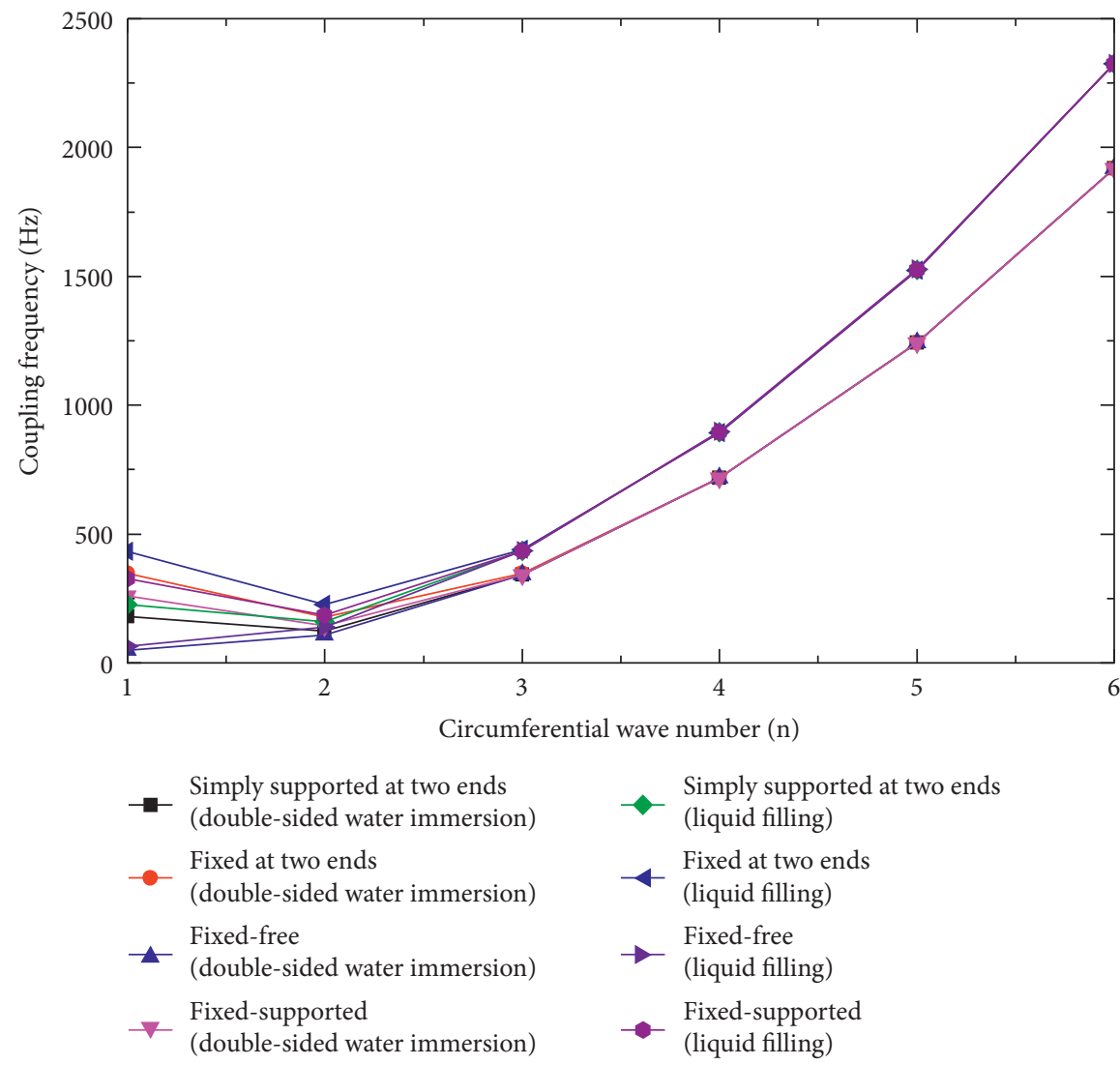

FiguRE 7: Coupling frequency curves of different boundary conditions.

shell with hydrostatic pressure, the coupling frequencies of liquid-filled ring-stiffened cylindrical shell, underwater ringstiffened cylindrical shell, and double-sided water immersion ring-stiffened cylindrical shell with the change of hydrostatic pressure were calculated. The calculation results of $(1,2)$ mode and $(1,3)$ mode are given in Figure 8.

It can be seen from the figure that when the hydrostatic pressure is $0 \mathrm{MPa}$, the coupling frequency of the liquid- filled ring-stiffened cylindrical shell is equal to that of the underwater ring-stiffened cylindrical shell, both of which are less than the coupling frequency of the double-sided water immersion ring-stiffened cylinder. With the increase of hydrostatic pressure, the coupling frequency of liquid-filled ring-stiffened cylindrical shell increases, the coupling frequency of underwater ring-stiffened cylindrical shell decreases, and the coupling frequency of 


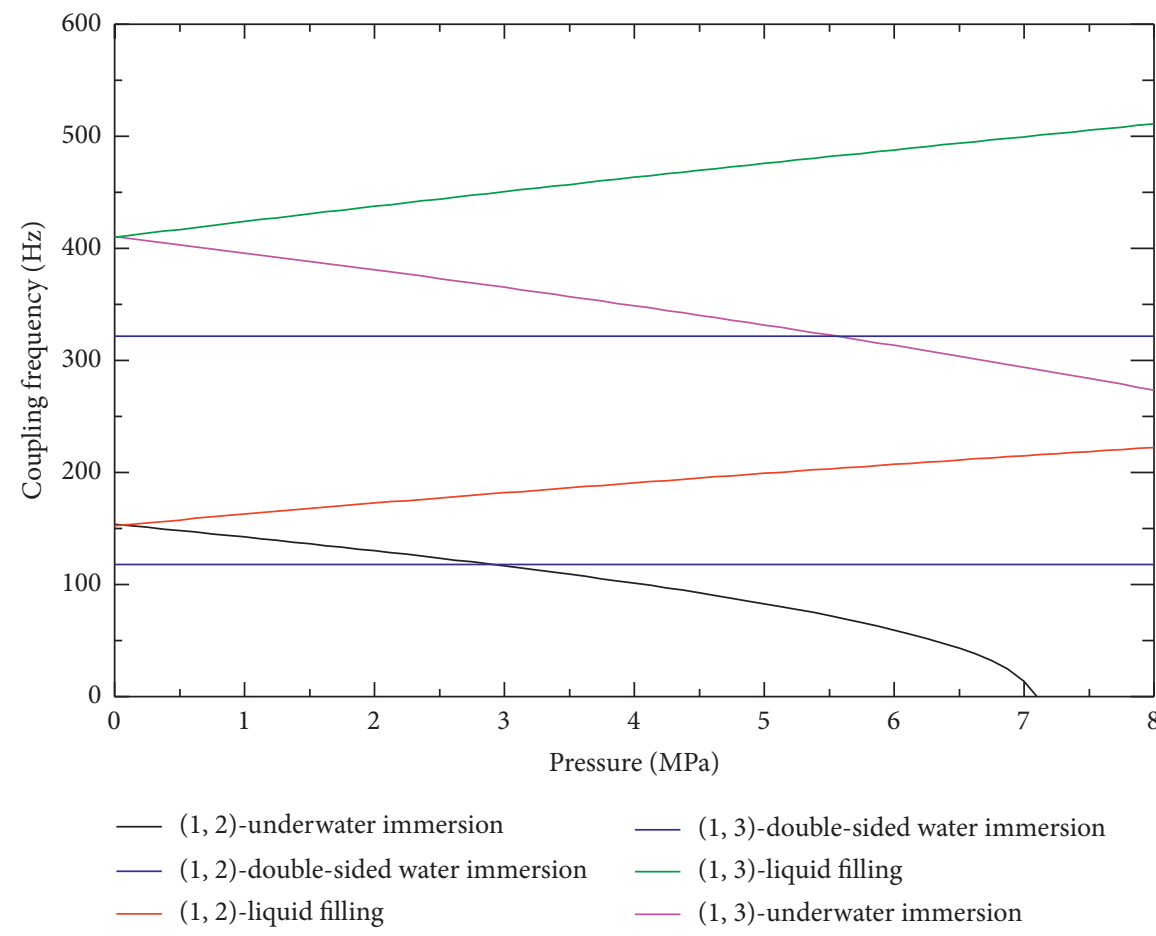

Figure 8: Coupling frequency curves of different hydrostatic pressure.

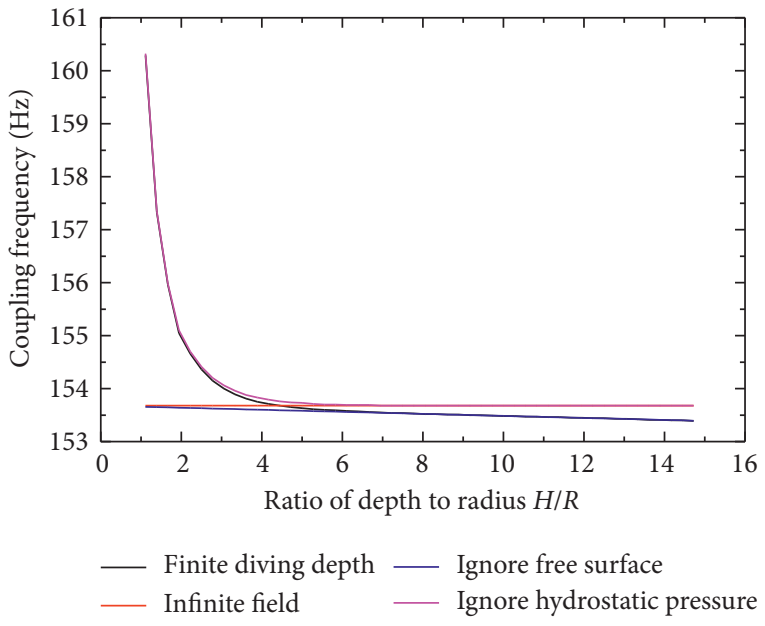

(a)

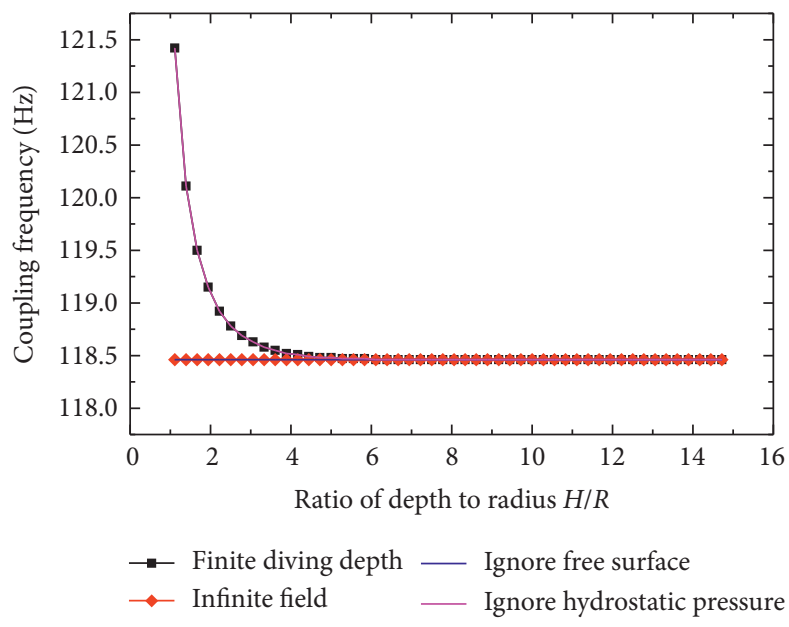

(b)

FIgURE 9: Coupling frequency curves of different diving depths. (a) Underwater immersion. (b) Water immersion on both sides.

double-sided water immersion ring rib cylindrical shell does not change. When the hydrostatic pressure increases to a certain value, the size of the coupling frequency of the underwater ring-stiffened cylindrical shell will exchange with that of the double-sided water immersion ringstiffened cylindrical shell. This shows that the effect of hydrostatic pressure is equivalent to changing the stiffness of the shell. The internal static pressure increases the stiffness of the shell, while the external static pressure reduces the stiffness of the shell. When the internal and external static pressures exist at the same time, the stiffness of the shell remains unchanged.
4.5. Influence of Free Surface. In order to study the influence of free surface on the coupling frequency of ring-stiffened cylindrical shell under the condition of finite diving depth, taking $(1,2)$ mode as an example, the variation curves of coupling frequency with hydrostatic pressure of submerged ring-stiffened cylindrical shell and double-sided water immersion ring-stiffened cylindrical shell under finite diving depth are given in Figure 9.

From the comparison curves in the figure, it can be seen that when near the free surface, the coupling frequency of submerged ring-stiffened cylindrical shell is greater than that of infinite domain, indicating that the free surface will 
increase the natural frequency of cylindrical shell. When the diving depth is far away from the free surface, the coupling frequency of submerged ring-stiffened cylindrical shell is smaller than that of infinite domain. In other words, when the diving depth of water is small, the influence of hydrostatic pressure on the coupling frequency of the shell is small, and the influence of free surface on the coupling frequency of the shell is greater, and the closer to the free surface, the higher the coupling frequency; when the diving depth of water is large, the influence of free surface on the coupling frequency of the shell can be ignored, and the greater the hydrostatic pressure, the smaller the coupling frequency.

It can be seen from Figure 9(b) that the free surface will also increase the coupling frequency of the double-sided water immersion ring-stiffened cylindrical shell. Since the hydrostatic pressure has no effect on the coupling frequency of the double-sided water immersion ring-stiffened cylindrical shell, the coupling frequency at finite diving depth coincides with that when hydrostatic pressure is ignored, and the coupling frequency of infinite domain coincides with that of neglecting free surface. From the above analysis, it can be concluded that the coupling frequency of the ringstiffened cylindrical shell will increase when it is close to the free surface, and the influence of the free surface can be ignored when the depth $H$ is greater than $5 R$.

\section{Conclusion}

(1) Without considering hydrostatic pressure, the coupling frequency of liquid-filled ring-stiffened cylindrical shell and submerged ring-stiffened cylindrical shell is equivalent, while the coupling frequency of double-sided water immersion ring-stiffened cylindrical shell is less than the former two.

(2) With the increase of the number of ribs and the height of ribs, the coupling frequency of doublesided water immersion cylindrical shell increases, and the effect of increasing the height of ribs is better than that of increasing the number of ribs.

(3) When the axial wave number is small, the boundary condition has a great influence on the frequency of double-sided water immersion ring-stiffened cylindrical shell; when the axial wave number is large, the influence of boundary conditions on the frequency can be ignored.

(4) The coupling frequency of liquid-filled ring-stiffened cylindrical shell increases with the increase of hydrostatic pressure, while the coupling frequency of submerged cylindrical shell decreases with the increase of hydrostatic pressure, and the coupling frequency of double-sided water immersion ring-stiffened cylindrical shell does not change with hydrostatic pressure.

(5) The coupling frequency of the underwater immersion ring-stiffened cylindrical shell and the doublesided water immersion ring-stiffened cylindrical shell will increase when it is close to the free surface, and the influence of the free surface can be ignored when the diving depth $H$ is greater than $5 R$.

\section{Appendix}




\section{Data Availability}

The data that support the findings of this study are available from the corresponding author upon reasonable request.

\section{Conflicts of Interest}

The authors declare that they have no conflicts of interest.

\section{Acknowledgments}

This study was supported by the National Natural Science Foundation of China (no. 52077218).

\section{References}

[1] Y. C. Zhang, W. Xu, Z. M. Li et al., "Dynamic characteristics analysis of marine propulsion shafting using multi-dof vibration coupling model," Shock and Vibration, vol. 2019, Article ID 5169156, 14 pages, 2019.

[2] X. M. Zhang, "Frequency analysis of submerged cylindrical shells with the wave propagation approach," International Journal of Mechanical Sciences, vol. 44, no. 7, pp. 1259-1273, 2002.

[3] S. A. Moeini, M. Rahaeifard, and M. T. Ahmadian, "Free vibration analysis. of functionally graded cylindrical shells stiffened by uniformly and non-uniformly distributed ring stiffeners," ASME International Mechanical Engineering Congress and Exposition, vol. 15, pp. 367-375, 2010.

[4] D. F. Yang, Research on Vibration of Ring-Stiffened Cylindrical Shell Filled with Fluid, Shanghai Jiao Tong University, Shanghai, China, 2003.

[5] M. Pourmahmoud, M. Salmanzadeh, and M. Mehrani, "Clamped-clamped boundary conditions for analysis free vibration of functionally graded cylindrical shell with a ring based on third order shear deformation theory," Engineering and Technology, vol. 41, pp. 684-689, 2010.

[6] R. Li, B. Liang, N. Noda et al., "Study on vibration of functionally graded cylindrical shells subjected to hydrostatic pressure by wave propagation method," Journal of Ship Mechanics, vol. 17, no. 1, pp. 148-154, 2013.

[7] M. M. Najafizadeh, A. Hasani, and P. Khazaeinejad, "Mechanical stability of functionally graded stiffened cylindrical shells," Applied Mathematical Modelling, vol. 33, no. 2, pp. 1151-1157, 2009.

[8] A. A. Jafari and M. Bagheri, "Free vibration of non-uniformly ring stiffened cylindrical shells using analytical, experimental and numerical methods," Thin-Walled Structures, vol. 44, no. 1, pp. 82-90, 2006.

[9] X. B. Li, H. Chen, and Z. Pan, "Study on dynamic characteristics of cylindrical shells filled with fluid," Ship Science and Technology, vol. 30, no. 2, pp. 45-50, 2008.

[10] X. Zhou, "Vibration and stability of ring-stiffened thin-walled cylindrical shells conveying fluid," Acta Mechanica Solida Sinica, vol. 25, no. 2, pp. 168-176, 2012.

[11] B. Liang, X. W. Liu, R. Li et al., "Study on vibration of fluidfilled cylindrical shells with ring-stiffener using wave propagation approach," Engineering Mechanics, vol. 33, no. 6, pp. 9-14, 2016.

[12] Z. Z. Liu, Characteristics of Power Flow and Sound Radiation in Cylindrical Shell-Fluid System Considering Hydrostatic Pressure, Huazhong University of Science\& Technology, Wuhan, China, 2009.
[13] C. Chen, T. Y. Li, X. Zhu et al., "Elastic pressure prediction of submerged ring-stiffened cylindrical shell based on frequency characteristics analysis," Ocean Engineering, vol. 32, no. 4, pp. 89-95, 2014.

[14] L. Cao, Y. Y. Ma, and Y. Y. Huang, "Free vibration of ringstiffened circular cylindrical shell with variable thickness," J. of HUST.(Urban Science Editon), vol. 24, no. 2, pp. 63-66, 2007.

[15] Z. Z. LIU, T. Y. LI, X. ZHU et al., "Effect of hydrostatic pressure on input flow in submerged ring-stiffened cylindrical shells," Journal of Ship Mechanics, vol. 15, no. 3, pp. 301-312, 2011.

[16] X. B. Li, "Study on free vibration analysis of circular cylindrical shells using wave propagation," Journal of Sound and Vibration, vol. 311, no. 3, pp. 667-682, 2008.

[17] X. M. Zhang, G. R. Liu, and K. Y. Lam, "Vibration analysis of thin cylindrical shells using wave propagation approach," Journal of Sound and Vibration, vol. 239, no. 3, pp. 397-403, 2001.

[18] X. B. Li, "Energy method for free vibration analysis of ringstiffened cylindrical shells," Journal of Ship Mechanics, vol. 5, no. 2, pp. 73-81, 2001.

[19] B. Liang, R. Li, X. W. Liu et al., "Coupled vibration of ringstiffened cylindrical shells subjected to hydrostatic pressure using wave propagation method," Journal of Vibration and Shock, vol. 33, no. 21, pp. 142-147, 2014.

[20] P. Wang, T. Y. Li, X. Zhu et al., "Frequency analysis of submerged cylindrical shell near the free surface," Journal of Vibration Engineering, vol. 5, pp. 772-778, 2016.

[21] P. Wang, T. Y. Li, X. Zhu et al., "Frequency analysis of a cylindrical shell immersed in shallow water," Shipbuilding of China, vol. 57, no. 3, pp. 72-82, 2016.

[22] P. Wang, T. Y. Li, X. Zhu et al., "Natural vibration characteristics analysis for an immersed cylindrical shell with a limited diving depth," Journal of Vibration and Shock, vol. 36, no. 15 , pp. 146-151, 2017.

[23] L. Gan, X. Li, and Z. Zhang, "Free vibration analysis of ringstiffened cylindrical shells using wave propagation approach," Journal of Sound and Vibration, vol. 326, no. 3-5, pp. 633-646, 2009.

[24] P. Wang, Vibro-acoustic Characteristics of a Finite Cylindrical Shell Submerged in Bounded Acoustic Media, Huazhong Unive-rsity of Science\&Technology, Wuhan, China, 2017. 